\title{
Exploring the Mental, Social and Lifestyle Effects of COVID-19 Infection on Syrian Refugees in Jordan: A Qualitative Study
}

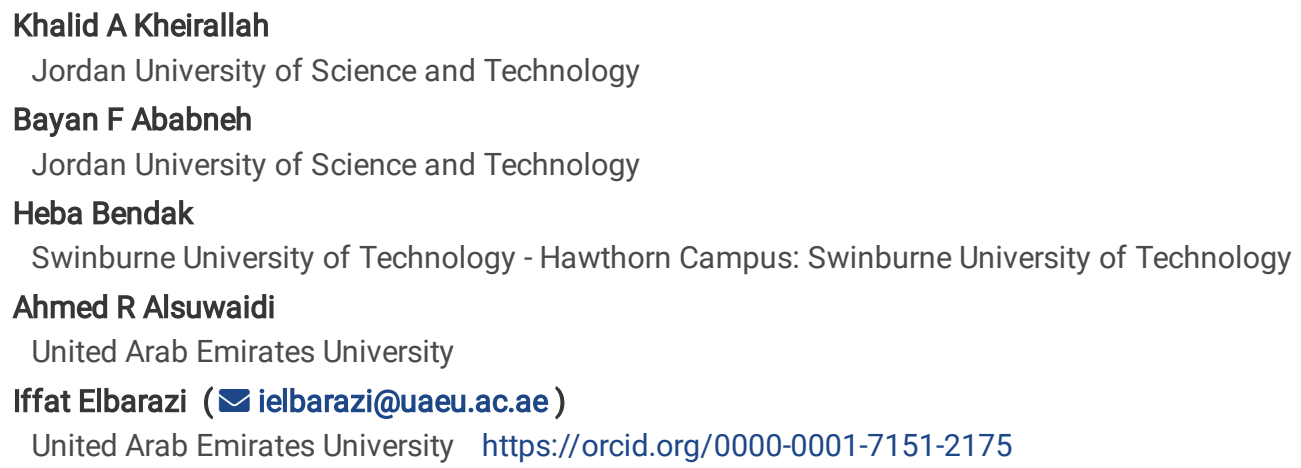

\section{Research}

Keywords: refugee, COVID-19, camps, community, healthcare, well-being

Posted Date: December 3rd, 2021

DOI: https://doi.org/10.21203/rs.3.rs-1101153/v1

License: (c) (i) This work is licensed under a Creative Commons Attribution 4.0 International License. Read Full License 


\section{Abstract}

Background: The fear of testing positive for COVID-19 infection has created panic among patients and families and discouraged some people from being tested and receiving medical care. Migrants and refugees are among the vulnerable populations that suffered disproportionately from the COVID-19 crisis. However, their experiences with COVID-19 positivity status have not been investigated. This study aimed to explore the physical, mental, and psychosocial impact of a positive COVID-19 diagnosis on Syrian refugees living in Jordan.

Methods: Using a qualitative approach, twenty phone interviews were conducted with ten adult Syrian refugees living within the camp and ten refugees living in non-camp (host-community) settings. A follow-up interviews with five health care providers in the refugee camp were conducted to explore the services and support given to the refugees with COVID-19 infection. The findings were thematically analysed using Braun \& Clarke's six-phase framework.

Results: Physical effects of a positive COVID-19 status varied according to the seriousness of the condition but affected most participants' lifestyle by adopting more precautionary measures and caring for their physical health. Self-isolation and fear to infect others were common themes identified among all participants living within community settings. Resorting to friends and family members for mental and psychological support was the only option available for all participants. Refugees living within the community preferred to manage their condition at home utilizing traditional remedies and avoided being tested for financial reasons, poor healthcare access and fear of being identified. Refugees living within camps had better access to testing, healthcare, and disease management and did not experience fear of being deported. They did not display worries and feelings of shame and guilt, which were feelings expressed by refugees living within the community. Both groups suffered mental and psychosocial health impacts, as well as social isolation and fear of death and disease complications.

Conclusions: COVID-19 infection has negatively impacted refugees' well-being with noticeable disparities across the different living conditions. While mental health impact seems to be standardized, refugees living within host-community may need more support into managing their condition, accessibility to free testing as well as treatment and healthcare services.

\section{Introduction}

More than 230 million people worldwide have been infected with severe acute respiratory syndrome coronavirus-2, the causative agent of the coronavirus Disease 2019 (COVID-19) as of October 2021(1). Initially centered in the Wuhan Province of China, the virus has spread globally and resulted in close to five million deaths across 215 countries(2). The clinical implications of the virus are variable and range between mild symptomology (flu-like symptoms) and severe respiratory distress and organ failure disease(3). Expectedly, health professionals have focused on the medical impacts of the virus while governments have focused on Non-Pharmaceutical Intervention (NPI) measures and vaccine administration when available. The NPI are public health measures that aim at infection containment using physical distancing and restrictions on travel, school, work, and other 'non-essential activities(4). While this is crucial for controlling the spread of the virus, research from previous pandemics has found that restrictive measures and perceived risk of infection resulted in negative impacts on psychological and social health such as depression, anxiety, posttraumatic stress disorder(5-7)' loneliness, isolation, anti-social behaviors, domestic violence, and child abuse $(8-10)$.

Preliminary research has found that the psychological and social health of vulnerable populations has been severely impacted as a result of COVID-19 related NPI measures (11-14). Yet, research studies that explored the specific challenges associated with a positive COVID-19 diagnosis in vulnerable populations are limited. Refugees are a particularly vulnerable group during the current pandemic due to their living conditions that increase their risk of being infected (15). For example, preventive health measures recommended to control the transmission of COVID-19, such as hand hygiene and physical distancing, are not possible to implement and/or sustain in highly dense and poorly maintained areas, such as refugee camp settings (16). Further, the majority of refugees currently reside under temporary living conditions within poor urban settings that suffer crowdedness, shortage of clean water and personal hygiene equipment. Shortage of public health services and financial means to support their families, and lack of proper sewage systems are additional challenges for the spread of COVID-19 among refugees. This increases their susceptibility to communicable, and non-communicable, diseases (17). Additionally, it may be more difficult for refugees to follow public health advice, as quarantining or self-isolating of COVID-19 positive cases can exacerbate the monetary challenges that refugees face on a daily basis(17). Such socio-economic burden may further contribute to spreading the infection within refugees' households and local communities.

In Jordan, a country that hosts around 664,000 registered and 500,000 unregistered Syrian refugees, the first cases of confirmed COVID-19 were reported in Zaatari and Azraq Refugee Camps in September 2020(18). While 80\% of Syrian refugees live within host-communities, outside of refugee camp settings, it is currently not known how many refugees outside of the camps have tested positive for COVID-19(18). The most recent report by the United Nations High Commissioner for Refugees (UNHCR) revealed that, as of March 8, 2021, approximately 2,400 refugees tested positive for COVID-19 inside refugee camps(18). During this period, the figure was below national average, as $2 \%$ of refugees tested 
positive, compared to $4 \%$ of the total population. However, this figure may have increased as Jordan had a spike in cases in late March 2021 , reaching the highest recorded number of cases per day (18).

Syrian refugees living in Jordan, within camp or host-community settings, suffer from economical disadvantages and poor living conditions as stated previously (19). COVID-19 infection and its related mitigation measures seemed to have complicated the situation and added fragilities to their living conditions. There are initial indications that refugees may refrain from seeking medical support due to fear of stigma associated with a positive COVID-19 diagnosis, including the guilt of contracting the virus and spreading it to others $(20,21)$. Aid workers in Rohingya camps in Bangladesh reported minimal testing amongst refugees with COVID-19 symptoms, as they feared isolation from their families, deportation, and even getting killed (20). Consequently, the social stigma associated with COVID-19 in general may result in symptom concealment, a delay in receiving early treatment, and a lower likelihood of compliance with public health measures (22). This may also result in clinically significant mental and social health effects. It is thus crucial to explore and assess the COVID-19-related social and psychological challenges specifically faced by refugees. This will help inform public health interventions and policies and curb the spread of the virus. In this study, we aim to purposely explore the physical, mental, and psychosocial impacts that a positive COVID-19 diagnosis has on Syrian refugees living in Jordan, within camp and host-community settings, utilizing a qualitative study design.

\section{Methods}

This study is reported according to the COnsolidated criteria for REporting Qualitative research (COREQ) (23). A qualitative approach was utilized using in-depth phone interviews using semi-structured interview guide. Snowballing sampling technique identified refugees who have had a COVID-19 infection between February 2020 and March 2021. A sample of 20 Syrian refugees, 10 males and 10 females, were verbally consented and interviewed. Ten of the refugees were living within refugee camp settings and rest were living within host-community setting. More than 10 refugees were approached but refused to participate due to different reasons ranging from lack of time to limited availability or simply being not interested. An interview guide was created to explore the potential impact under investigation. Additionally, we interviewed five healthcare professionals who were directly involved with the healthcare of refugees within camps to explore the management, services and support for positive COVID-19 cases during the crisis. The interviews were conducted in April 2021. Five health care providers (HCP) (3 Medical doctors and nurses from Al-Zaatari camp) were interviewed over the phone by the same investigator who conducted the interviews with the participants. Participants were approached through snowball technique.

\section{Study Design Theoretical framework}

An interview guide was created based on (Lazarus and Folkman 1984) coping strategy model(24) and (Burr \& Klein 1994) conceptual framework of coping strategies(25).. The principal investigator (PI); (I.E) developed the interview guide (Supplementary file 1). The other research team members reviewed the interview guide and agreed on the major questions and prompts. Ethical approval was obtained from the Institutional Review Board of Jordan University of Science and Technology (24/139/2021).

\section{Participant selection}

\section{Sampling}

Purposive and convenience sample of 20 refugees with recovered COVID-19 infection, and five health care providers (HCP) from Zaatari camp, were included in the study.

\section{Method approach}

Participants were approached by phone and WhatsApp and, if consented, were interviewed over the phone and included in the study. All interviews were audio-recorded and then transcribed into verbatim text.

\section{Interviewer characteristics}

$\mathrm{IE}$, an experienced qualitative researcher and the study $\mathrm{PI}$, trained a research assistant (BFA) via zoom on how to conduct interviews and on thematic analysis and identifying theme. BFA holds a Master of Public Health, IE holds a DrPH in qualitative research methods. IE has supervised the entire process of the study.

\section{Participants}


Participants were first identified through workers within healthcare facility services provided to refugees within both settings. Once potential participants were identified, they were contacted by phone. Upon initial approval, participants received the study information package (via WhatsApp) to review and provide initial agreement to participate. Upon receiving selected participants' agreements, participants were contacted to schedule a phone interview. None of the participants were known to the interviewer. Participants who did not provide consent were not further contacted. Only adult participants who self-reported to ever been diagnosed with and recovered from COVID-19 infection between February and April 2021 were interviewed. Around 30 participants were invited but only 20 accepted to conduct the interview. Similarly, HCP were approached by the same worker who helps in identifying refugees. We planned from the beginning to interview 5 HCP to explore the services and support provided for positive COVID-19 patients in the camp. The study information package shared with participants included information about study aims, voluntary participation, confidentiality, and nature of questions to be asked along with a statement that personal information will not be collected. During the scheduled phone interview, a verbal consent was secured, and participants were asked to identify potential study participants. Each interview lasted between 40-60 min.

\section{Data collection}

\section{Interview guide}

The interview guide was developed in both Arabic and English with questions exploring the mental, social, psychological, spiritual and lifestyle impact of a positive COVID-19 on participants. The interview guide was reviewed by the research team who agreed on the final questions and concepts to be addressed through interviews. Interviews were conducted by trained researchers in qualitative interviews in Arabic. All interviews were recorded then transcribed after obtaining verbal consent from participants. A research assistant collected all recordings, transcribed them, and translated them from Arabic to English. All interviews were imported to NVivo 12 for analysis and coding.

\section{Data saturation}

Data saturation was reached after interviewing five participants from each group; however, we decided to keep interviewing participants to ensure we have a wide variety and more representation of the targeted refugees' population.

\section{Reflexivity and Bias}

To ensure researchers reflexivity and to reduce bias, some of the interviews' transcripts were returned to participants by WhatsApp for review and approval. Participants showed no concerns and were satisfied with the transcribed words. The PI and the research assistant reviewed and verified the transcripts.

\section{Health care providers Interviews}

Semi-structured phone interviews were conducted. Each interview lasted for 30-40 minutes, with five health care workers (three physicians and two nurses) using open ended questions that covered mainly: management protocols for COVID-19 cases, classification of cases, the available vaccines, the availability of vaccines, Polymerase Chain Reaction (PCR) testing, dealing with suspected cases, availability of medical services, coverage by insurance, refugees' behavior toward following safety precautions and contacting confirmed cases.

\section{Data Analysis}

Framework thematic analysis was performed. A IE and BFA have revised the transcripts of the interview for triangulation. IE and BFA extracted themes individually then agreed on final themes with other research team members.

\section{Description of the coding tree}

The interview guide was divided into questions related to physical, social, mental, lifestyle and spiritual effects of a COVID- 19 positive diagnosis on the participant. The coding was based on these five main codes.

\section{Derivation of themes}

Thematic analysis using Braun and Clarke six steps process was performed. Themes derivation was based on the reported effects as described by participants. Physical themes were based on symptoms experienced by participants. Social themes included the impact on social relations during the infection period with direct family, members and friends. Mental health themes included the impact on psychological status during and post COVID-19 diagnosis and returning to normal life. Lifestyle themes included impact on use of dietary supplements, diet modifications, sleep and exercise. Spiritual themes included impact on relationship with God, beliefs and faith, and views toward death and diseases.

\section{Results}

Ten refugees living in one camp (Al-Zaatari camp, Jordan) and 10 living within host-community settings were included in the analysis. The participants' ages ranged from 20 to 56 years and $70 \%$ were females (Table 1). Themes and subthemes were organized based on the main 
aims of the study. For each major theme and emerging themes are included in (Table 2 \& Table3.). 
Table 1

Participants' Demographics

\begin{tabular}{|c|c|c|c|c|c|c|c|c|c|}
\hline Participant & $\begin{array}{l}\text { Age } \\
\text { (years) }\end{array}$ & Gender & Education & Occupation & $\begin{array}{l}\text { Marital } \\
\text { status }\end{array}$ & $\begin{array}{l}\text { Place of } \\
\text { residence }\end{array}$ & $\begin{array}{l}\text { Co- } \\
\text { habitants }\end{array}$ & $\begin{array}{l}\text { Arrival } \\
\text { date to } \\
\text { Jordan }\end{array}$ & $\begin{array}{l}\text { Financial } \\
\text { source }\end{array}$ \\
\hline Refugee01F & 56 & Female & School & Housewife & Married & $\begin{array}{l}\text { Irbid- } \\
\text { outside } \\
\text { camp }\end{array}$ & $\begin{array}{l}\text { Daughters } \\
\text { and } \\
\text { husband }\end{array}$ & $\begin{array}{l}2012,3 \\
\text { years } \\
\text { later left } \\
\text { the camp } \\
\text { to Irbid } \\
\text { rented } \\
\text { house }\end{array}$ & $\begin{array}{l}\text { A } 50 \\
\text { Jordanian } \\
\text { Dinars (JDs) } \\
\text { monthly } \\
\text { food coupon } \\
\text { from } \\
\text { UNHCR, } \\
\text { approximate } \\
\text { salary by her } \\
\text { husband } \\
\text { around } 200 \\
\text { JDS }\end{array}$ \\
\hline $\begin{array}{l}\text { Refugee } \\
01 \mathrm{M}\end{array}$ & 31 & Male & High school & $\begin{array}{l}\text { Ex- } \\
\text { mechanic }\end{array}$ & Single & $\begin{array}{l}\text { Irbid- } \\
\text { outside } \\
\text { camp }\end{array}$ & $\begin{array}{l}\text { Five } \\
\text { single } \\
\text { brothers }\end{array}$ & $\begin{array}{l}2012 \\
\text { inside } \\
\text { camp, } \\
\text { then } \\
\text { moved to } \\
\text { Irbid } \\
\text { rented } \\
\text { house }\end{array}$ & $\begin{array}{l}\text { My five } \\
\text { brothers } \\
\text { working to } \\
\text { cove life } \\
\text { needs } \\
\text { approximate } \\
\text { income } \\
\text { between } \\
500-700 \\
\text { JDS }\end{array}$ \\
\hline $\begin{array}{l}\text { Refugee } \\
\text { 02F }\end{array}$ & 51 & Female & $\begin{array}{l}\text { University/bachelor } \\
\text { degree in law }\end{array}$ & Housewife & Married & $\begin{array}{l}\text { Irbid- } \\
\text { outside } \\
\text { camp }\end{array}$ & $\begin{array}{l}\text { Husband, } \\
\text { son and } \\
\text { daughter }\end{array}$ & $\begin{array}{l}2015 \\
\text { came to } \\
\text { Amman } \\
\text { then } \\
\text { moved to } \\
\text { Irbid } \\
\text { rented } \\
\text { house }\end{array}$ & $\begin{array}{l}\text { Husband } \\
\text { working with } \\
\text { good salary } \\
\text { approximate } \\
\text { salary } \\
\text { around } 200 \\
\text { JDS and } \\
300 \text { JDS }\end{array}$ \\
\hline $\begin{array}{l}\text { Refugee } \\
\text { 02M }\end{array}$ & 25 & Male & $\begin{array}{l}\text { Studying the } \\
\text { English language } \\
\text { at a private } \\
\text { university }\end{array}$ & Waiter & Single & $\begin{array}{l}\text { Irbid- } \\
\text { outside } \\
\text { camp }\end{array}$ & $\begin{array}{l}\text { Parents, } \\
\text { one sister } \\
\text { and one } \\
\text { brother }\end{array}$ & $\begin{array}{l}2012 \\
\text { stayed at } \\
\text { camp for } \\
\text { one year } \\
\text { then } \\
\text { moved to } \\
\text { Irbid } \\
\text { rented } \\
\text { house }\end{array}$ & $\begin{array}{l}\text { Father is } \\
\text { working as a } \\
\text { private } \\
\text { English } \\
\text { teacher, and } \\
\text { mother sells } \\
\text { homemade } \\
\text { pastries } \\
\text { Approximate } \\
\text { Income } \\
\text { around } 250 \\
\text { JDS and } \\
350 \text { JDS }\end{array}$ \\
\hline $\begin{array}{l}\text { Refugee } \\
\text { O3F }\end{array}$ & 25 & Female & $\begin{array}{l}\text { University/bachelor } \\
\text { degree in Finance }\end{array}$ & Secretory & Single & $\begin{array}{l}\text { Irbid- } \\
\text { outside } \\
\text { camp }\end{array}$ & $\begin{array}{l}\text { Live with } \\
\text { parents } \\
\text { and two } \\
\text { brothers }\end{array}$ & $\begin{array}{l}2012, \\
\text { then } \\
\text { moved } \\
\text { from } \\
\text { camp to } \\
\text { rented } \\
\text { house in } \\
\text { Irbid at } \\
2015\end{array}$ & $\begin{array}{l}\text { Father is the } \\
\text { driver, and I } \\
\text { am } \\
\text { secretory } \\
\text { Approximate } \\
\text { income } \\
\text { around } 250 \\
\text { and } 350 \\
\text { JDS }\end{array}$ \\
\hline $\begin{array}{l}\text { Refugee } \\
\text { 03M }\end{array}$ & 45 & Male & School & Casher & Married & $\begin{array}{l}\text { Irbid- } \\
\text { outside } \\
\text { camp }\end{array}$ & $\begin{array}{l}\text { Live with } \\
\text { wife and } \\
\text { three } \\
\text { children }\end{array}$ & $\begin{array}{l}\text { In } 2013 \\
\text { direct to } \\
\text { Irbid } \\
\text { rented } \\
\text { house }\end{array}$ & $\begin{array}{l}50 \text { jds } \\
\text { monthly } \\
\text { food coupon } \\
\text { from UNHCR } \\
\text { Approximate } \\
\text { salary } \\
\text { between } 150 \\
\text { and } 200 \\
\text { JDS }\end{array}$ \\
\hline
\end{tabular}




\begin{tabular}{|c|c|c|c|c|c|c|c|c|c|}
\hline Participant & $\begin{array}{l}\text { Age } \\
\text { (years) }\end{array}$ & Gender & Education & Occupation & $\begin{array}{l}\text { Marital } \\
\text { status }\end{array}$ & $\begin{array}{l}\text { Place of } \\
\text { residence }\end{array}$ & $\begin{array}{l}\text { Co- } \\
\text { habitants }\end{array}$ & $\begin{array}{l}\text { Arrival } \\
\text { date to } \\
\text { Jordan }\end{array}$ & $\begin{array}{l}\text { Financial } \\
\text { source }\end{array}$ \\
\hline $\begin{array}{l}\text { Refugee } \\
\text { 04F }\end{array}$ & 45 & Female & High school & Housewife & Married & $\begin{array}{l}\text { Irbid- } \\
\text { outside } \\
\text { camp }\end{array}$ & $\begin{array}{l}\text { Live with } \\
\text { husband } \\
\text { and } \\
\text { daughter }\end{array}$ & $\begin{array}{l}2012, \\
\text { then } \\
\text { moved } \\
\text { from } \\
\text { camp to } \\
\text { rented } \\
\text { house in } \\
\text { lrbid } 6 \\
\text { months } \\
\text { later }\end{array}$ & $\begin{array}{l}\text { Husband is } \\
\text { working as a } \\
\text { porter and } \\
\text { receiving a } \\
\text { monthly } \\
50 \text { jds food } \\
\text { coupon } \\
\text { from UNHCR } \\
\text { Approximate } \\
\text { income } 150 \\
\text { JDS }\end{array}$ \\
\hline $\begin{array}{l}\text { Refugee } \\
04 M\end{array}$ & 49 & Male & High school & Painter & Married & $\begin{array}{l}\text { Irbid- } \\
\text { outside } \\
\text { camp }\end{array}$ & $\begin{array}{l}\text { Live with } \\
\text { wife, son } \\
\text { and two } \\
\text { daughters }\end{array}$ & $\begin{array}{l}2013, \\
\text { then } \\
\text { moved } \\
\text { from } \\
\text { camp to } \\
\text { rented } \\
\text { house in } \\
\text { Irbid a } \\
\text { few } \\
\text { months } \\
\text { later }\end{array}$ & $\begin{array}{l}\text { Only work is } \\
\text { the financial } \\
\text { source } \\
\text { Approximate } \\
\text { income } 150 \\
\text { to } 250 \text { JDS }\end{array}$ \\
\hline $\begin{array}{l}\text { Refugee } \\
05 F\end{array}$ & 20 & Female & $\begin{array}{l}\text { Studying nutrition } \\
\text { at university }\end{array}$ & Student & Single & $\begin{array}{l}\text { Irbid- } \\
\text { outside } \\
\text { camp }\end{array}$ & $\begin{array}{l}\text { With } \\
\text { mother } \\
\text { and } \\
\text { brother }\end{array}$ & $\begin{array}{l}\text { Came } \\
\text { from } \\
\text { Turkey in } \\
2016 \text { and } \\
\text { bought a } \\
\text { house in } \\
\text { Irbid }\end{array}$ & $\begin{array}{l}\text { Our financial } \\
\text { status is } \\
\text { perfect } \\
\text { Approximate } \\
\text { income } \\
\text { more than } \\
500 \text { JDS per } \\
\text { month }\end{array}$ \\
\hline $\begin{array}{l}\text { Refugee } \\
05 \mathrm{M}\end{array}$ & 39 & Male & High school & Porter & Married & $\begin{array}{l}\text { Irbid- } \\
\text { outside } \\
\text { camp }\end{array}$ & $\begin{array}{l}\text { With wife } \\
\text { and } \\
\text { daughter }\end{array}$ & $\begin{array}{l}\text { Lived in } \\
\text { al Zaatari } \\
\text { camp } \\
\text { from } \\
2012 \text { to } \\
2015 \\
\text { then } \\
\text { moved to } \\
\text { a rented } \\
\text { house in } \\
\text { Irbid }\end{array}$ & $\begin{array}{l}\text { Approximate } \\
\text { income } \\
\text { between } 150 \\
\text { to } 200 \text { JDS }\end{array}$ \\
\hline $\begin{array}{l}\text { Refugee } \\
\text { 06F }\end{array}$ & 38 & Female & $\begin{array}{l}\text { Bachelor degree in } \\
\text { management }\end{array}$ & Receptionist & Married & $\begin{array}{l}\text { Almafraq- } \\
\text { inside } \\
\text { camp }\end{array}$ & $\begin{array}{l}\text { Husband } \\
3 \text { sons } \\
\text { and three } \\
\text { daughters }\end{array}$ & $\begin{array}{l}\text { Almafraq- } \\
\text { inside } \\
\text { camp } \\
\text { since } \\
2012\end{array}$ & $\begin{array}{l}\text { I received a } \\
\text { monthly } 50 \\
\text { JDS food } \\
\text { coupon and } \\
\text { 20JD for } \\
\text { each one of } \\
\text { my family } \\
\text { members by } \\
\text { UNCHR in } \\
\text { addition to } \\
\text { my job } \\
\text { Approximate } \\
\text { income } 260 \\
\text { JDS }\end{array}$ \\
\hline
\end{tabular}




\begin{tabular}{|c|c|c|c|c|c|c|c|c|c|}
\hline Participant & $\begin{array}{l}\text { Age } \\
\text { (years) }\end{array}$ & Gender & Education & Occupation & $\begin{array}{l}\text { Marital } \\
\text { status }\end{array}$ & $\begin{array}{l}\text { Place of } \\
\text { residence }\end{array}$ & $\begin{array}{l}\text { Co- } \\
\text { habitants }\end{array}$ & $\begin{array}{l}\text { Arrival } \\
\text { date to } \\
\text { Jordan }\end{array}$ & $\begin{array}{l}\text { Financial } \\
\text { source }\end{array}$ \\
\hline \multirow[t]{2}{*}{$\begin{array}{l}\text { Refugee } \\
06 \mathrm{M}\end{array}$} & 37 & Male & $\begin{array}{l}\text { Diploma of } \\
\text { management }\end{array}$ & $\begin{array}{l}\text { Security } \\
\text { guard }\end{array}$ & Married & $\begin{array}{l}\text { Almafraq- } \\
\text { inside } \\
\text { camp }\end{array}$ & With wife & $\begin{array}{l}\text { Almafraq- } \\
\text { inside } \\
\text { camp } \\
\text { since } \\
2014\end{array}$ & $\begin{array}{l}\text { I am } \\
\text { working, and } \\
\text { we are } \\
\text { receiving a } \\
\text { monthly } 50 \\
\text { JDS food } \\
\text { coupon and } \\
\text { 20JD for } \\
\text { each one of } \\
\text { family } \\
\text { members by } \\
\text { UNCHR }\end{array}$ \\
\hline & & & & & & & & & $\begin{array}{l}\text { Approximate } \\
\text { income } 140 \\
\text { JDS }\end{array}$ \\
\hline \multirow[t]{2}{*}{$\begin{array}{l}\text { Refugee } \\
\text { 07F }\end{array}$} & 42 & Female & High school & Housewife & Married & $\begin{array}{l}\text { Almafraq- } \\
\text { inside } \\
\text { camp }\end{array}$ & $\begin{array}{l}\text { With } \\
\text { husband } \\
\text { and three } \\
\text { sons and } \\
\text { two } \\
\text { daughters }\end{array}$ & $\begin{array}{l}\text { Almafraq- } \\
\text { inside } \\
\text { camp } \\
\text { since } \\
2013\end{array}$ & $\begin{array}{l}\text { Are receiving } \\
\text { a monthly } \\
50 \text { JDS food } \\
\text { coupon and } \\
20 \mathrm{JD} \text { for } \\
\text { each one of } \\
\text { a family } \\
\text { member by } \\
\text { UNCHR }\end{array}$ \\
\hline & & & & & & & & & $\begin{array}{l}\text { Approximate } \\
\text { income } 140 \\
\text { JDS }\end{array}$ \\
\hline \multirow[t]{2}{*}{$\begin{array}{l}\text { Refugee } \\
07 \mathrm{M}\end{array}$} & 43 & Male & Diploma of nursing & $\begin{array}{l}\text { Coordinator } \\
\text { of the clinic } \\
\text { inside the } \\
\text { camp }\end{array}$ & Married & $\begin{array}{l}\text { Almafraq- } \\
\text { inside } \\
\text { camp }\end{array}$ & $\begin{array}{l}\text { With a } \\
\text { wife and } \\
\text { four } \\
\text { daughters }\end{array}$ & $\begin{array}{l}\text { Almafraq- } \\
\text { inside } \\
\text { camp } \\
\text { since } \\
2012\end{array}$ & $\begin{array}{l}\text { Receiving a } \\
\text { monthly } 50 \\
\text { JDS food } \\
\text { coupon and } \\
\text { 20JD for } \\
\text { each one of } \\
\text { a family } \\
\text { member by } \\
\text { UNCHR }\end{array}$ \\
\hline & & & & & & & & & $\begin{array}{l}\text { Approximate } \\
\text { income } 220 \\
\text { JDS }\end{array}$ \\
\hline $\begin{array}{l}\text { Refugee } \\
08 \mathrm{~F}\end{array}$ & 29 & Female & High school & $\begin{array}{l}\text { Distribution } \\
\text { of health } \\
\text { brochures }\end{array}$ & Married & $\begin{array}{l}\text { Almafraq- } \\
\text { inside } \\
\text { camp }\end{array}$ & $\begin{array}{l}\text { With } \\
\text { husband } \\
\text { and one } \\
\text { son and } \\
\text { two } \\
\text { daughters }\end{array}$ & $\begin{array}{l}\text { Almafraq- } \\
\text { inside } \\
\text { camp } \\
\text { since } \\
2014\end{array}$ & $\begin{array}{l}\text { Are receiving } \\
\text { a monthly } \\
50 \text { JDS food } \\
\text { coupon and } \\
20 \mathrm{JD} \text { for } \\
\text { each one of } \\
\text { a family } \\
\text { member by } \\
\text { UNCHR } \\
\text { approximate } \\
\text { income } 200 \\
\text { JDS }\end{array}$ \\
\hline \multirow[t]{2}{*}{$\begin{array}{l}\text { Refugee } \\
08 \mathrm{M}\end{array}$} & 45 & Male & High school & Barber & Married & $\begin{array}{l}\text { Almafraq- } \\
\text { inside } \\
\text { camp }\end{array}$ & $\begin{array}{l}\text { With a } \\
\text { wife and } \\
\text { nine } \\
\text { daughters }\end{array}$ & $\begin{array}{l}\text { Almafraq- } \\
\text { inside } \\
\text { camp } \\
\text { since } \\
2012\end{array}$ & $\begin{array}{l}\text { Receiving a } \\
\text { monthly } 50 \\
\text { JDS food } \\
\text { coupon and } \\
\text { 20JD for } \\
\text { each one of } \\
\text { a family } \\
\text { member by } \\
\text { UNCHR }\end{array}$ \\
\hline & & & & & & & & & $\begin{array}{l}\text { Approximate } \\
\text { income } 320 \\
\text { JDS }\end{array}$ \\
\hline
\end{tabular}




\begin{tabular}{|c|c|c|c|c|c|c|c|c|c|}
\hline Participant & $\begin{array}{l}\text { Age } \\
\text { (years) }\end{array}$ & Gender & Education & Occupation & $\begin{array}{l}\text { Marital } \\
\text { status }\end{array}$ & $\begin{array}{l}\text { Place of } \\
\text { residence }\end{array}$ & $\begin{array}{l}\text { Co- } \\
\text { habitants }\end{array}$ & $\begin{array}{l}\text { Arrival } \\
\text { date to } \\
\text { Jordan }\end{array}$ & $\begin{array}{l}\text { Financial } \\
\text { source }\end{array}$ \\
\hline \multirow[t]{2}{*}{$\begin{array}{l}\text { Refugee } \\
\text { O9F }\end{array}$} & 35 & Female & High school & Housewife & Married & $\begin{array}{l}\text { Almafraq- } \\
\text { inside } \\
\text { camp }\end{array}$ & $\begin{array}{l}\text { With } \\
\text { husband } \\
\text { and two } \\
\text { sons and } \\
\text { two } \\
\text { daughters }\end{array}$ & $\begin{array}{l}\text { Almafraq- } \\
\text { inside } \\
\text { camp } \\
\text { since } \\
2013\end{array}$ & $\begin{array}{l}\text { Are receiving } \\
\text { a monthly } \\
50 \text { JDS food } \\
\text { coupon and } \\
20 \mathrm{JD} \text { for } \\
\text { each one of } \\
\text { a family } \\
\text { member by } \\
\text { UNCHR }\end{array}$ \\
\hline & & & & & & & & & $\begin{array}{l}\text { Approximate } \\
\text { income } 220 \\
\text { JDS }\end{array}$ \\
\hline $\begin{array}{l}\text { Refugee } \\
09 \mathrm{M}\end{array}$ & 25 & Male & $\begin{array}{l}\text { Bachelor degree in } \\
\text { marketing }\end{array}$ & $\begin{array}{l}\text { Security } \\
\text { guard }\end{array}$ & Married & $\begin{array}{l}\text { Almafraq- } \\
\text { inside } \\
\text { camp }\end{array}$ & $\begin{array}{l}\text { With a } \\
\text { wife and } \\
\text { one } \\
\text { daughter }\end{array}$ & $\begin{array}{l}\text { Almafraq- } \\
\text { inside } \\
\text { camp } \\
\text { since } \\
2013\end{array}$ & $\begin{array}{l}\text { Receiving a } \\
\text { monthly } 50 \\
\text { JDS food } \\
\text { coupon and } \\
20 J D \text { for } \\
\text { each one of } \\
\text { a family } \\
\text { member by } \\
\text { UNCHR } \\
\text { approximate } \\
\text { income } 160 \\
\text { JDS }\end{array}$ \\
\hline \multirow[t]{2}{*}{$\begin{array}{l}\text { Refugee } \\
\text { O10F }\end{array}$} & 24 & Female & School & Housewife & Married & $\begin{array}{l}\text { Almafraq- } \\
\text { inside } \\
\text { camp }\end{array}$ & $\begin{array}{l}\text { With } \\
\text { husband } \\
\text { and two } \\
\text { sons }\end{array}$ & $\begin{array}{l}\text { Almafraq- } \\
\text { inside } \\
\text { camp } \\
\text { since } \\
2013\end{array}$ & $\begin{array}{l}\text { Receiving a } \\
\text { monthly } 50 \\
\text { JDS food } \\
\text { coupon and } \\
\text { 20JD for } \\
\text { each one of } \\
\text { a family } \\
\text { member by } \\
\text { UNCHR }\end{array}$ \\
\hline & & & & & & & & & $\begin{array}{l}\text { Approximate } \\
\text { income } 180 \\
\text { JDS }\end{array}$ \\
\hline $\begin{array}{l}\text { Refugee } \\
010 \mathrm{M}\end{array}$ & 45 & Male & High school & Porter & Married & $\begin{array}{l}\text { Almafraq- } \\
\text { inside } \\
\text { camp }\end{array}$ & $\begin{array}{l}\text { With a } \\
\text { wife and } \\
\text { three } \\
\text { sons, and } \\
\text { one } \\
\text { daughter }\end{array}$ & $\begin{array}{l}\text { Almafraq- } \\
\text { inside } \\
\text { camp } \\
\text { since } \\
2012\end{array}$ & $\begin{array}{l}\text { Receiving a } \\
\text { monthly } 50 \\
\text { JDS food } \\
\text { coupon and } \\
20 J D \text { for } \\
\text { each one of } \\
\text { a family } \\
\text { member by } \\
\text { UNCHR } \\
\text { approximate } \\
\text { income 220 } \\
\text { JDS }\end{array}$ \\
\hline
\end{tabular}

Table 2

Major Themes

\begin{tabular}{|lll|}
\hline Number & Major Themes & Sub-themes \\
\hline $\begin{array}{l}\text { Theme } \\
\text { one }\end{array}$ & Physical effect & Symptoms, hospitalization and ability to cope with physical symptoms \\
$\begin{array}{l}\text { Theme } \\
\text { Two }\end{array}$ & $\begin{array}{l}\text { Mental and psychological } \\
\text { impact }\end{array}$ & Anxiety, anger, Stigma and fear \\
\hline $\begin{array}{l}\text { Theme } \\
\text { Three }\end{array}$ & Social impact & Isolation and exclusion as well as social bonding and extra support. \\
\hline $\begin{array}{l}\text { Theme } \\
\text { Four }\end{array}$ & Spiritual impact & $\begin{array}{l}\text { Increase in spiritual practices and closer relation to God, fate and destiny, belief in God's will, } \\
\text { and coping mechanism }\end{array}$ \\
\hline $\begin{array}{l}\text { Theme } \\
\text { five }\end{array}$ & lifestyle and behavior & Healthier lifestyle, increased infection control precautions, and strengthening immune system. \\
\hline
\end{tabular}


The physical impact of a positive COVID-19 diagnosis on the refugees, within camp and host community settings, was not different except that those in the camp had better medical support and access for testing and for medications. Most of the participants in the camp had a mild to moderate COVID-19 disease. None of them required hospitalization. However, those in host communities expressed the fact that they did not seek medical assistance due to financial reasons although they had symptoms. For example, one participant did not perform the COVID-19 test despite having symptoms.

She said: "I had symptoms like my other family members but preferred not to get tested to save money. I did not need to go to hospital or receive a medical treatment."

Those in the camp had access to medical services, including medications, which reduced their anxiety and their dealing with the experienced symptoms. Most of the participants in both settings indicated experiencing flu-like symptoms, body aches and pain, headache, and loss of taste and smell. Few indicated that the taste of smell did not last for long while some stated that their concerns that they did not regain their original sense of smell and had an unpleasant smell most of the time.

\section{Theme Two}

The mental and psychological impact of the COVID-19 positive status was similar between both groups in terms of the stigma and fear of being identified as a positive case while most expressed their anger, anxiety and fear of complications especially those who had COVID-19 during the earlier period of the pandemic. Anger was more common among those who were afraid to lose their job and access to work being casual workers who depend on their daily income. Moreover, some expressed their fear and anxiety originating from their fear of losing their spouse or children because of COVID-19.

A participant from the camp said, "I am annoyed because I lost my job from the date, I got infected. My elder brother is giving me pocket money. I hope to find a new job soon". Another participant from the community said "I was upset and sad because I lost my salary during the isolation period and my wife borrowed money from her cousin to cover some daily needs".

Similarly, two participants expressed their frustration:

"At the beginning, I was annoyed because I lost my salary since I need money to help my family in daily life needs and university fees and to save some to get married, but thanks God I returned back to my job"

"I was so sad that getting the virus forced me to borrow money from neighbors to buy food"

For others, worrying about losing their loved ones was more frustrating. For example, one of them said:

"I was worried about my father and unfortunately we lost him."

Another said:

"I was worried only about my parents. They lost their smell and taste sensations. When I get my salary this month inshallah, I will refer them to the medical advice"

Stigma was experienced by some in the camp and was a deterrent for seeking help for some in the community.

A participant from the camp said:

"Here at camp, people are closed minded and consider who got the virus being unclean person with poor hygiene".

Another in the community indicated that:

"I did not want people to know that my daughter and I had COVID. I preferred to keep away from the neighbors so they do not know about our positive infection".

\section{Theme Three}

The social impact among those in the refugee camp varied. Some specified that they did not get much support from spouse especially in the household chores. One lady said that she was even mistreated by her husband:

"My husband was not supportive, and he got mad with me. He had to take care of the home and kids .... He did not really look after them properly.... Actually, I didn't receive good family support like any other infected persons and that made me sad ....and I was crying all the time". 
Many of those in the camps and in the host-community said that they resorted to phone calls to their family members and friend in Jordan or Syria to get their support and to spend their isolation time. Some used spiritual practices, reading, studying, internet and television to cope with the situation. Some expressed their over protection to children, husband by isolating themselves and by providing them with herbal and homemade remedies to avoid complications. Some indicated that the COVID-19 period helped them to reconnect with family members and strengthened their bonds.

A lady from the camp said:

"I had more time during isolation to talk to my sisters in Syria over phone, this is an advantage of being isolated"

"In the first 2 days of my isolation, I spent time on Facebook and WhatsApp. Then when my parents and brothers got the infection, I was cooking and cleaning the house"

"The Father of my daughter's friend was calling us all the time and provided all support and advised us to use vitamins $C$ and $D$ ".

\section{Another said "I was busy doing my homework and studying" Theme Four}

Most participants considered COVID-19 to have a positive impact on their spirituality. Many expressed their views that the experience made them closer to God and that they spent most of their isolation time praying, reading Quran and self-reflecting on their lives. However, so many of the participants stated that their spiritual practices and relation with God was always strong and that the COVID-19 did not impact on their beliefs and faith. Here are some related quotes:

"We are Muslims and do our prayers all the time, but when I got the result, I prayed to God for protecting my family and me more".

"I had more time to listen to Quran and thanks to God, I am Muslim and do my prayers on time, and I have a good relationship with God".

"I spent my isolation reading Quran"/

"We are Muslims, and we are close to God".

"We are Muslims, and we are close to God, but after getting the virus, I felt that I had to do prayers on time and to get closer to God..".

\section{Theme Five}

More than half of participants inside and outside the camp believed that COVID-19 was an actual situation and not a man-made conspiracy, while others thought the virus was man-made. Most participants acknowledged the importance of preventive measures, a healthy immune system, and homemade remedies.

"We are reminding each other about following the safety precautions strictly; we are afraid of each other of getting the virus again. May God protect us and all Muslims around the world."

"My husband and I were calling each other all the time checking our health status,and thank God my family didn't get the virus."

"This virus was made at the laboratory to get rid of elderly people in the world, and don't ask me why because this is my inner feeling."

"It's real and transmitted all over the world".

"We usually follow a healthy lifestyle even before the COVID-19; nothing changed."

\section{Emerging Themes (Table.3)}


Table 3

Emerging Themes Reported from Qualitative Assessment of the Impact of COVID-19 on Syrian Refugees

\begin{tabular}{|ll|}
\hline Setting & \\
\hline Camp & Host Community \\
\hline Poor household support & Economical and financial impact \\
\hline Social Exclusion & Fear of loss of financial support \\
\hline Stigma & Limited access to medical care \\
\hline Loss of Job & Closer and tightened social relationship within the same household \\
\hline Poor social support & \\
\hline
\end{tabular}

Emerging themes were classified based on being in camp or host community settings (Table 2). Those who were living in the community had a financial and economic impact on COVID-19. So many of them specified that the financial implications affected their access to testing, medical help, and coping ability. They experienced anger and fear for losing their job and the possibility to lose their income. Mothers or females in this group had a strong feeling of responsibility to look after the souse being the breadwinner and the children.

Those in the camp experienced more social exclusion, social issues with husbands and neighbors affecting their mental health and social relationships. Those in the refugee camp had no problems in terms of access to medical care and medicine. They had no fear to be out of their jobs; only one or two mentioned this issue and some mentioned being affected financially.

"I received free treatment and received free coupons with 20 Jordanian Dinars for each one of us from UNCHR in the camp."

"Here at camp, the environment is not healthy and closed-minded also, and they are not educated; they considered who got the virus that he is a dirty person or with scabies."

"I am not upset due to not getting paid; as I told you, we are receiving help from UNCHR and live for free at camp, and our water and electricity are free."

"I didn't get my salary for the 14 isolation days, but that doesn't matter since I received free treatment and receiving free coupons with 20JDs for each one of us from UNCHR."

"My husband was mad because he lost his salary for 14 days the duration of isolation and was not able to buy cigarettes, but now everything is good, we didn't have any effects on our daily life needs because it is free in the camp."

Those outside the camp discussed the impact of the virus on their financial situation too. one participant said:

"I preferred to isolate myself at home using home remedies to save costs for buying bread or chicken for her daughters".

Another referred to her job loss, saying:

"I lost my job from the date I got infected by the virus; my big brother is giving me pocket money; I want to search for a new job."

Health Care Providers interview findings

Health care providers reported that they are following updated protocol for COVID-19 treatment according to World Health Organization (WHO) and Jordanian Ministry of Health ( $\mathrm{MOH}$ ) protocols and according to pandemic status in Jordan. They all claimed that refugees in Al Zaatari camp are receiving the best and free treatment for COVID-19 and even other diseases. They reported that at the beginning of the pandemic, the infected cases with COVID-19 were isolated in the Dead Sea area like any other case before an isolation area was prepared at the camps sites. They also reported that PCR tests are free and available all times for suspected cases; symptomatic or asymptomatic. Once a case is confirmed, it is classified according to severity (mild, moderate or severe). Mild and moderate cases are treated in the camp and severe cases are referred to hospitals outside the camp. Treatment, which mainly depends on the severity, mainly include administering antipyretics, antibiotics, oxygen masks, and/or nebulizers. Interviewed HCPs also reported that the vaccination campaigns were initiated as that within Jordan using the same vaccines for Jordanians (Sinopharm, Pfizer and newly added AstraZeneca), and that all medical services for COVID-19 cases and vaccinations in the Zaatari camp are provided free of charge. They all agreed upon the easier tracking system in the camp for close contacts of confirmed cases. However, they also agreed that some of refugees hide their symptoms to avoid testing, isolation, and losing their jobs and some hide to avoid embarrassment and stigma. Even some of infected cases chose to be isolated at home because they have children with disabilities, the health care team in the camp visited them and provided them with medical and needed support. 


\section{Discussion}

The living conditions of Syrian refugees in Jordan are not optimal as the country does not support re-settlement programs. Uncertainties associated with Syrian refugees are expected to be further complicated by the spread of COVID-19 and mitigation measures. This has added to the disease's economic, social, and mental health burden among such vulnerable groups. It is necessary to investigate the overlapping fragilities associated with COVID-19 infection among Syrian refugees to reduce the associated health burdens. The current study explored the physical, mental and psychosocial impacts of a positive COVID-19 cases on Syrian refugees living within the camp and host-community settings in Jordan utilizing a qualitative approach. The results indicated a significant level of economic, social, and mental health impacts expressed by participants associated with a positive COVID-19 infection. Impacts presented in this study seems not to be uniform among refugees and to differ by settings (in camp vs. host-community). Public health interventions should consider such differential burdens and focus on reducing the social stigma associated with COVID-19 and the economic barriers associated with such disease.

The impacts of COVID-19 on Syrian refugees living in Jordan seem to overlap and interconnect and may not be addressed separately. Physical effects, for example, intersected with fear, social stigma and economic burden, which may have negatively affected social support( 26,27$)$. A COVID-19 infection for a refugee indicates loss of a temporary, low paying, job and a significant reduction to ones earning potential and ability to meet essential needs(27). This significant stressor adds to the fragility of living conditions of refugees and exposes them to a burden that was not expected. This is mainly for refugees within host communities, as they still have to pay rent and provide daily living essentials. In contrast, camp refugees have access to more humanitarian aid and do not pay rent. Moreover, refugees in the camps had better access to health care services as reported by participants. This is supported by El-Khatib et al. (2020) who argue that the Zaatari camp, adapted many supportive mental health activities to support refugees during COVID pandemic(28).

Availability of healthcare services within camp settings seems to buffer the physical impact by eliminating the financial needs required to secure testing and medical expenses. This is even though humanitarian funding has been reduced due to the global effects of COVID-19. Still, Jordan was one of the countries that included refugees in its national response to COVID-19, including testing, care, and immunization (28) .

While there is a global call for more attention to the mental and psychological impact of COVID-19, refugees seem to be more vulnerable to such impact given their prior exposure to conflict-related trauma(29). Anxiety, anger, and fear among refugees are well-established given the levels of traumatic exposure they witnessed in Syria, during the refugee journey, and while living as a refugee(29). This adds to the burden of mental health problems among refugees and puts them at higher risk of long-term impact(29-31). Of note, as well, is that this impact seems to be universal regardless of the settings (camp vs. host-community). Surveillance, screening and fine-tuned interventions for the effects of COVID-19 may be a critical need to avoid the potential adverse impacts of complicated, intersected, stressors on mental health wellbeing(32)

The social impact seems to be rooted within the Middle Eastern culture, as a family is the center of attention from caregivers. Families in such culture provide social support to those in need and ensure providing attention when needed. Alzoubi, found pre- COVID-19 era, that Syrian refugees had different coping strategies with other predictors(31) The authors found that seeking social support was associated with "being female, older, and widowed; having a lower education and lower total income; being dissatisfied with their income; being non-employed and having chronic illnesses"(31). Gender roles also seemed to emerge during COVID-19 as wives did not perceive the social support from their husbands during the COVID-19 infection period. Regardless, the social dimension of COVID-19 infection may have presented times for refugees to cope and provide social support when needed. At the same time, isolation and exclusion relate more to the perceived stigma associated with COVID-19 infection and has been the case among refugees and at the global level. The United Nation Women Jordan Rapid Assessment Report that tensions within households were reported by refugees in camps as well as economic hardships(33).

Social stigmatization of COVID-19 infected individuals seems to have potentially flourished with dramatic stories in media and on the internet(34-36). Worldwide, several features of Stigma toward individuals from Asian descent, those with recent travel history, and healthcare professionals have also been reported ${ }^{3(37)}$. Still, no study reported on COVID-19 related Stigma among vulnerable refugee groups. Social Stigma associated with COVID-19 infection may be attributed to the disease being new and not fully perceived among the public. In Jordan, the first cases of COVID-19 were severely stigmatized through being labelled, stereotyped, discriminated against, and treated separately. This stigmatization was augmented by the lack of accurate information and the widespread misinformation related to the disease. This infodemic $(2,34)$ became a global phenomenon that may have complicated the social acceptance of the disease and presented it as a reason for stereotyping and discrimination against those infected or who came made a contact with the infected individuals. Accordingly, it is expected that Syrian refugees being stigmatized due to COVID-19 infection seek immediate healthcare services less frequently and be discouraged from adopting health behaviors to prevent the spread of the disease. This calls for showing more empathy to COVID-19 patients and ensuring that the public understands the disease itself and facilitating the adoption of measures to help infected cases keep themselves and their loved ones safe and infection-free. While this may not be feasible given the living conditions of refugees within camps or host communities, self-isolation and community support for quarantines could be a solution using social norms and local health experts from within the refugee population. The public should also be informed about the potential role of stigmatization on individuals and the community and the need to have them 
play a role in preventing discrimination through kindness, speaking up against negative stereotypes, learning more about mental health, and sharing individual experiences to provide the support needed. Caregivers and local leaders working with refugees should also encourage dispelling fears, misconceptions and the Stigma associated with COVID-19. As necessary, healthcare providers should consider social Stigma among refugees as a significant mental health problem that could be exacerbated by the socioeconomic hardship caused by the pandemic. This will complicate refugees' integration and increase their living uncertainties.

\section{Limitation And Strengths}

This is the first study to explore the impact of a positive COVID -19 infection on the wellbeing of Syrian refugees within camp and host community settings. It is the first to explore views, beliefs, and positive or negative impacts of such infection in a holistic approach. Limitations include using the phone for the interviews instead of face-to-face, which was due to the COVID-19 mitigation measures. As in qualitative studies, generalization beyond the study participants and small sample size are still an issue. More studies on a larger scale would provide a better picture with less risk of bias. A longitudinal study approach may also facilitate assessing the impact of COVID-19 on the long run and fine-tune interventions to avoid the potential life-long implications of the possible effects on the refugees.

\section{Conclusion}

A COVID -19 positive infection had various influences and effects on Syrian refugees in Jordan. Living within the camp or host-community settings can be considered an essential determinant of how a positive COVID-19 diagnosis may impact the health and wellness of a Syrian refugee. Support for those who test positive from local authorities and UNCHR is highly needed to reduce the burden on this population and their families.

\section{Abbreviations}

NPI

Non-Pharmaceutical Intervention

UNHCR

United Nations High Commissioner for Refugees

$\mathrm{HCP}$

Health care providers

PCR

Polymerase chain reaction

WHO

World Health Organization

Ministry of Health

$\mathrm{MOH}$

\section{Declarations}

Ethics approval: This study was performed in line with the principles of the Declaration of Helsinki. Ethical approval was granted by the Institutional Review Board of Jordan University of Science and Technology (24/139/2021).

Consent to participate: Informed verbal consent was obtained from all individual participants included in the study.

Consent for publication: Authors are responsible for the correctness of the statements provided in the manuscript.

Availability of data and material: Upon request to the corresponding author.

Competing Interest: The authors have no conflicts of interest to declare .

Funding: The authors did not receive support from any organization for the submitted work.

Authors' contributions: IE, KAK and BFA conceptualized the study. BFA conducted the interviews, and IE and BFA analyzed the data. IE, BFA, KAK analyzed and agreed on the themes. IE, BFA, HB, KAK wrote and reviewed the manuscript. ARA critically revised the manuscript for important intellectual content. All authors approved the final manuscript.

\section{Acknowledgements}


The authors would like to acknowledge all participants and the Faculty of Research at Jordan University of Science and Technology for their support of this study.

\section{References}

1. John Hopkins COVID-19 Map - Johns Hopkins Coronavirus Resource Center 2021 [Accessed October 102021

2. (WHO) WHO. WHO CoronavirusDisease Dashboard 2021 [Available from: https://covid19.who.int/ Accessed October 102021

3. Struyf T, Deeks JJ, Dinnes J, Takwoingi Y, Davenport C, Leeflang MM, et al. Signs and symptoms to determine if a patient presenting in primary care or hospital outpatient settings has COVID-19 disease. Cochrane Database Syst Rev. 2020;7(7):Cd013665.

4. Duarte Alonso A, Kok SK, Bressan A, O'Shea M, Sakellarios N, Koresis A, et al. COVID-19, aftermath, impacts, and hospitality firms: An international perspective. Int J Hosp Manag. 2020;91:102654.

5. Hawryluck L, Gold WL, Robinson S, Pogorski S, Galea S, Styra R. SARS control and psychological effects of quarantine, Toronto, Canada. Emerg Infect Dis. 2004;10(7):1206-12.

6. Reynolds DL, Garay JR, Deamond SL, Moran MK, Gold W, Styra R. Understanding, compliance and psychological impact of the SARS quarantine experience. Epidemiol Infect. 2008;136(7):997-1007.

7. Charania NA, Tsuji LJ. Assessing the effectiveness and feasibility of implementing mitigation measures for an influenza pandemic in remote and isolated First Nations communities: a qualitative community-based participatory research approach. Rural Remote Health. 2013;13(4):2566.

8. Ott M, Shaw SF, Danila RN, Lynfield R. Lessons learned from the 1918-1919 influenza pandemic in Minneapolis and St. Paul, Minnesota. Public health reports (Washington, DC: 1974). 2007;122(6):803-10.

9. John N, Casey SE, Carino G, McGovern T. Lessons Never Learned: Crisis and gender-based violence. Developing World Bioethics. 2020;20(2):65-8.

10. Lin L, Savoia E, Agboola F, Viswanath K. What have we learned about communication inequalities during the H1N1 pandemic: a systematic review of the literature. BMC Public Health. 2014;14:484

11. Chu IY, Alam P, Larson HJ, Lin L. Social consequences of mass quarantine during epidemics: a systematic review with implications for the COVID-19 response. J Travel Med. 2020;27(7).

12. Torales J, O'Higgins M, Castaldelli-Maia JM, Ventriglio A. The outbreak of COVID-19 coronavirus and its impact on global mental health. Int J Soc Psychiatry. 2020;66(4):317-20.

13. Brooks S, Webster LS, et al. The psychological impact of quarantine and how to reduce it: rapid review of the evidence

14. The Lancet. 2020; 395 pp. 912-20

15. Brooks SK, Smith LE, Webster RK, Weston D, Woodland L, Hall I, et al. The impact of unplanned school closure on children's social contact: rapid evidence review. Euro Surveill. 2020;25(13).

16. Beech H, Hubbard B. Unprepared for the

17. worst: world's most vulnerable brace for virus. The New York Times. 2020

18. N. S. Distancing is impossible': refugee camps race to avert coronavirus catastrophe.. Nature [Internet]. 2020; 05:[581(7806):18

p.].Accessed November 52021

19. Kassem II. Refugees besieged: The lurking threat of COVID-19 in Syrian war refugee camps. Travel Med Infect Dis. 2020;38:101736.

20. (UNHCR) UHCfR. UNHCR Jordan Fact Sheet February 2021 [Available from:

https://reporting.unhcr.org/sites/default/files/Jordan\%20country\%20factsheet\%20-\%20February\%202021.pdf [Accessed July 28 2021.

21. Tiltnes ÅA, Zhang H, Pedersen J. The living conditions of Syrian refugees in Jordan.: Fafo Oslo.; 2019 [

22. Saifee J, Franco-Paredes C, Lowenstein SR. Refugee Health During COVID-19 and Future Pandemics. Curr Trop Med Rep. 2021:1-4.

23. Truelove S, Abrahim O, Altare C, Lauer SA, Grantz KH, Azman AS, et al. The potential impact of COVID-19 in refugee camps in Bangladesh and beyond: A modeling study. PLoS Med. 2020;17(6):e1003144.

24. Alemi Q, Stempel C, Siddiq H, Kim E. Refugees and COVID-19: achieving a comprehensive public health response. Bull World Health Organ. 2020;98(8):510-A.

25. Tong A, Sainsbury P, Craig J. Consolidated criteria for reporting qualitative research (COREQ): a 32-item checklist for interviews and focus groups. Int J Qual Health Care. 2007;19(6):349-57.

26. RS L, S. F. Stress, appraisal, and coping.. New York: Springer; 1984.

27. W.R B, S.R K. Reexamining Family Stress: New Theory and Research. London: Sage Publications; 1994. 
28. Ren SY, Gao RD, Chen YL. Fear can be more harmful than the severe acute respiratory syndrome coronavirus 2 in controlling the corona virus disease 2019 epidemic. World J Clin Cases. 2020;8(4):652-7.

29. Organization for Economic Cooperation and Development O, (ILO). ILO. The impact of the COVID-19 pandemic on jobs and

30. incomes in G20 economies 2021 [Available from: https://www.ilo.org/wcmsp5/groups/public/--dgreports/cabinet/documents/publication/wcms_756331.pdf.

31. El-Khatib Z, Al Nsour M, Khader YS, Abu Khudair M. Mental health support in Jordan for the general population and for the refugees in the Zaatari camp during the period of COVID-19 lockdown. Psychological Trauma: Theory, Research, Practice, and Policy. 2020;12(5):511-4.

32. Fouad FM, McCall SJ, Ayoub H, Abu-Raddad LJ, Mumtaz GR. Vulnerability of Syrian refugees in Lebanon to COVID-19: quantitative insights. Conflict and Health. 2021;15(1):13.

33. Mhaidat NM, Al-Husein BA, Alzoubi KH, Hatamleh DI, Khader Y, Matalqah S, et al. Knowledge and Awareness of Colorectal Cancer Early Warning Signs and Risk Factors among University Students in Jordan. J Cancer Educ. 2016.

34. Alzoubi FA, Ali RA, Al-Gharaibeh AH. Resettled Syrian refugees in Jordan: Survival or health promotion. Nursing Open. 2021;8(1):273-83.

35. Søvold LE, Naslund JA, Kousoulis AA, Saxena S, Qoronfleh MW, Grobler C, et al. Prioritizing the Mental Health and Well-Being of Healthcare Workers: An Urgent Global Public Health Priority. Frontiers in Public Health. 2021;9:514.

36. Jordan UNW. Rapid assessment On the impacts of covid-19. 2020 [Available from: https://scihub.ru/https://www.ncbi.nlm.nih.gov/pmc/articles/PMC7538065/.

37. (WHO) WHO. The COVID-19 Infodemic 2021 [Available from: https://www.who.int/health-topics/infodemic/the-covid-19infodemic\#tab=tab_1.

38. Eysenbach G. Infodemiology and infoveillance: framework for an emerging set of public health informatics methods to analyze search, communication and publication behavior on the Internet. J Med Internet Res. 2009;11(1):e11.

39. (WHO). WHO. Managing the covid-19 Infodemic: Promoting healthy behaviors and mitigating the harm from misinofrmation and disinformation 20202 [Available from: https://www.who.int/news/item/23-09-2020-managing-the-covid-19-infodemic-promoting-healthybehaviours-and-mitigating-the-harm-from-misinformation-and-disinformation.

40. Prevention CfDCa. Reducing Stigma 2021 [Available from: https://www.cdc.gov/mentalhealth/stress-coping/reduce-stigma/index.html? CDC_AA_refVal=https\%3A\%2F\%2Fwww.cdc.gov\%2Fcoronavirus\%2F2019-ncov\%2Fdaily-life-coping\%2Freducing-stigma.html.

\section{Figures}

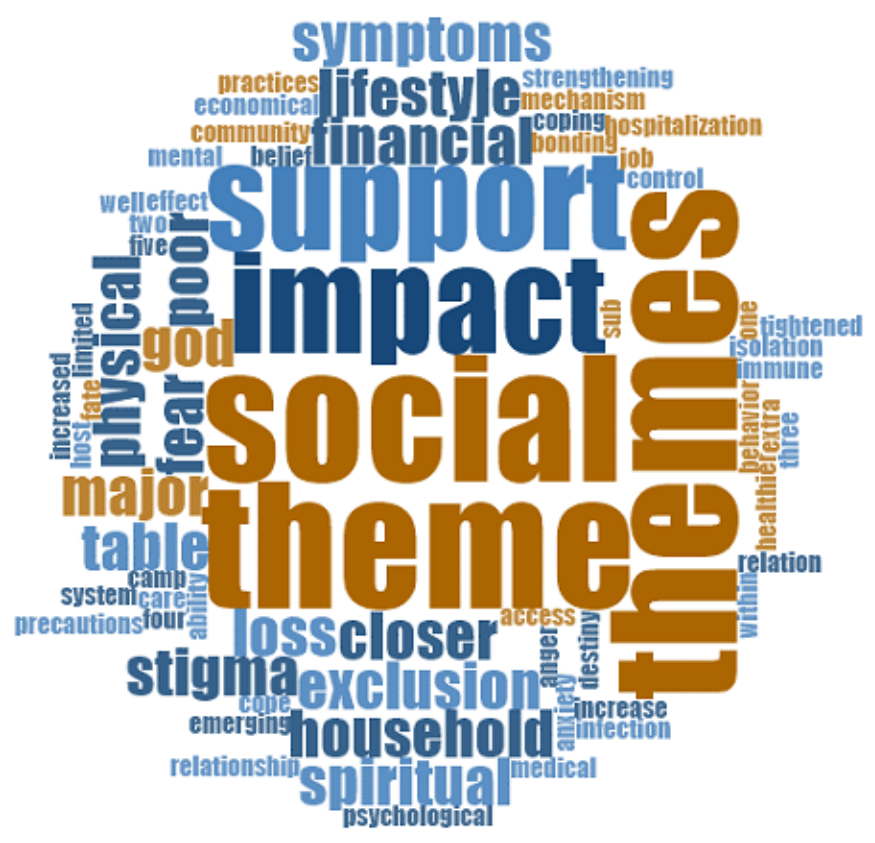

Figure 1

Represents most the common words repeated and used by the participants

Page $16 / 17$ 


\section{Supplementary Files}

This is a list of supplementary files associated with this preprint. Click to download.

- EffectsofCOVIDinterviewguide.pdf 